\title{
Antiferromagnetic magnons as highly squeezed Fock states underlying quantum correlations
}

\author{
Akashdeep Kamra $\odot,{ }^{1, *}$ Even Thingstad, ${ }^{1}$ Gianluca Rastelli, ${ }^{2,3}$ Rembert A. Duine, ${ }^{4,5,1}$ Arne Brataas, ${ }^{1}$ \\ Wolfgang Belzig, ${ }^{2}$ and Asle Sudb $\emptyset^{1}$ \\ ${ }^{1}$ Center for Quantum Spintronics, Department of Physics, Norwegian University of Science and Technology, NO-7491 Trondheim, Norway \\ ${ }^{2}$ Department of Physics, University of Konstanz, D-78457 Konstanz, Germany \\ ${ }^{3}$ Zukunftskolleg, University of Konstanz, D-78457 Konstanz, Germany \\ ${ }^{4}$ Institute for Theoretical Physics, Utrecht University, 3584CC Utrecht, The Netherlands \\ ${ }^{5}$ Department of Applied Physics, Eindhoven University of Technology, Eindhoven, The Netherlands
}

(Received 8 April 2019; revised manuscript received 27 August 2019; published 5 November 2019)

\begin{abstract}
Employing the concept of two-mode squeezed states from quantum optics, we demonstrate a revealing physical picture for the antiferromagnetic ground state and excitations. Superimposed on a Néel ordered configuration, a spin-flip restricted to one of the sublattices is called a sublattice magnon. We show that an antiferromagnetic spin-up magnon is composed of a quantum superposition of states with $n+1$ spin-up and $n$ spin-down sublattice magnons and is thus an enormous excitation despite its unit net spin. Consequently, its large sublattice spin can amplify its coupling to other excitations. Employing von Neumann entropy as a measure, we show that the antiferromagnetic eigenmodes manifest a high degree of entanglement between the two sublattices, thereby establishing antiferromagnets as reservoirs for strong quantum correlations. Based on these insights, we outline strategies for exploiting the strong quantum character of antiferromagnetic (squeezed) magnons and give an intuitive explanation for recent experimental and theoretical findings in antiferromagnetic magnon spintronics.
\end{abstract}

DOI: 10.1103/PhysRevB.100.174407

\section{INTRODUCTION}

As per the Heisenberg uncertainty principle, the quantum fluctuations of two noncommuting observables cannot simultaneously be reduced to zero. However, it is possible to generate a state with the quantum noise in one observable reduced below its ground-state limit at the expense of enhanced fluctuations in the other observable [1,2]. Considering a single mode or frequency of light, such states, generally called squeezed vacuum [1,2], have proven instrumental in the detection of gravitational waves [3] with a sensitivity beyond the quantum ground-state limit [4-6]. Furthermore, squeezed vacuum states have applications in quantum information [7-11] since they exhibit quantum correlations and entanglement. These are best represented and exploited via the two-mode squeezed vacuum states, where the two participating modes are entangled and correlated [1]. The widely studied [1,2] single- and two-mode squeezed vacuums may be considered a special case, corresponding to zero photon number(s), of a wider class: squeezed Fock states [12,13]. While investigated theoretically, the latter have been largely forgotten, probably owing to the experimental challenge of generating them. The squeezing concept applies to bosonic modes in general, and squeezed states of magnons [14-17] and phonons [18] have also been achieved experimentally.

The concept of squeezed Fock states $[12,13]$ has proven valuable in understanding the spin excitations of ordered magnets $[19,20]$. Squeezed magnons have been shown to be

\footnotetext{
*akashdeep.kamra@ntnu.no
}

the eigenexcitations of a ferromagnet [19,21]. A squeezed magnon is composed of a coherent superposition of the different odd-number states of the spin-1 magnon [19,20,22]. This bestows it a noninteger average spin larger than 1 . The relatively weak spin-nonconserving interactions, such as dipolar fields and crystalline anisotropy, underlie the magnon squeezing in ferromagnets. These spin-nonconserving interactions were further found to result in two-sublattice magnets hosting excitations with spin varying continuously between positive and negative values [20]. In contrast, exchange interaction in a two-sublattice magnet leads to a strong squeezing effect, which does not affect the excitation spin and forms a main subject of the present paper. Being eigenexcitations, squeezed magnons are qualitatively distinct in certain ways from the squeezed states of light discussed above, which are nonequilibrium states generated via an external drive. At the same time, the two kinds of states share several similar features on account of their wave functions being mathematically related. To emphasize this difference, we employ terminology in which "squeezed state of a boson" refers to a nonequilibrium state, while a "squeezed boson" is an eigenmode [23].

Instigated by recent experimental breakthroughs [24-29], interest in antiferromagnets (AFMs) for practical applications has been invigorated [30-34]. Due to the well-known strong quantum fluctuations in AFMs, they have also been the primary workhorse of the quantum magnetism community [35]. The Néel ordered configuration, which is consistent with most of the experiments, is not the true quantum ground state of an AFM. Furthermore, quantum fluctuations destroy any order in a one-dimensional isotropic AFM. These and related general ideas applied to AFMs bearing geometrically 


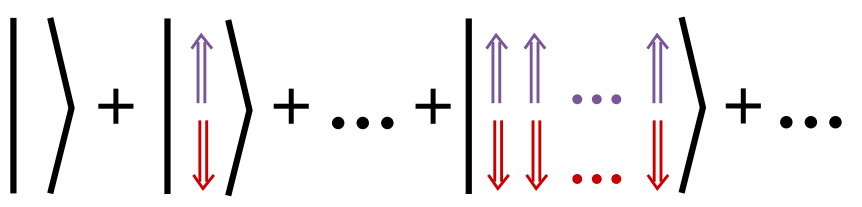

(a)

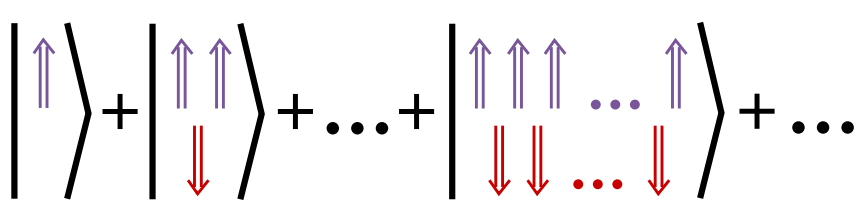

(b)

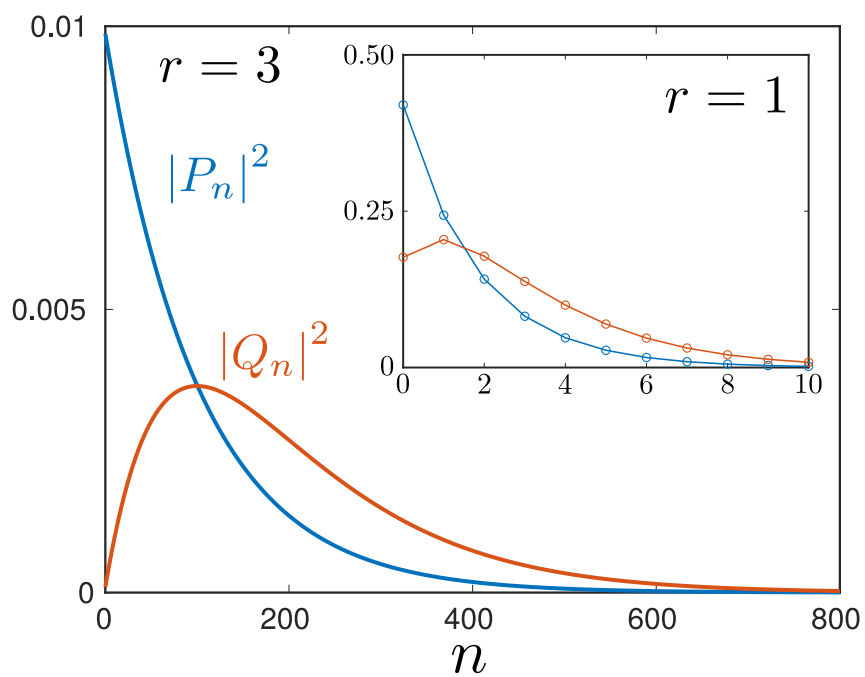

(c)

FIG. 1. Schematic depiction of spatially uniform antiferromagnetic (a) vacuum and (b) spin-up eigenmodes. (a) The vacuum mode, represented as $|0\rangle_{\mathrm{sq}}=\sum_{n} P_{n}|n, n\rangle_{\text {sub }}$, is a superposition over states with an equal number of spin-up and -down sublattice magnons. (b) The spin-up squeezed magnon, represented as $|\uparrow\rangle_{\mathrm{sq}}=$ $\sum_{n} Q_{n}|n+1, n\rangle_{\text {sub }}$, is composed of states with one extra spin-up sublattice magnon. (c) Squared amplitudes corresponding to the sublattice-magnon states constituting the uniform squeezed vacuum and spin-up eigenmodes for squeeze parameters of 3 (main) and 1 (inset).

frustrated interactions underlie quantum spin liquids [36-38], which are devoid of order in the ground state and host exotic, topologically nontrivial excitations embodying massive entanglement.

We here develop the squeezing picture for the ground state and excitations of a simple, two-sublattice AFM. It continuously connects and allows a unified understanding of classical and quantum as well as ordered and disordered antiferromagnetic states. We show that the AFM eigenmodes are obtained by pairwise, two-mode squeezing of sublattice magnons, the spin-1 excitations delocalized over one of the two sublattices. Focusing on spatially uniform modes, the antiferromagnetic ground state is a superposition of states with an equal number of spin-up and -down sublattice magnons [Figs. 1(a) and 1(c)]. The result is a net spin on each sublattice diminished by an

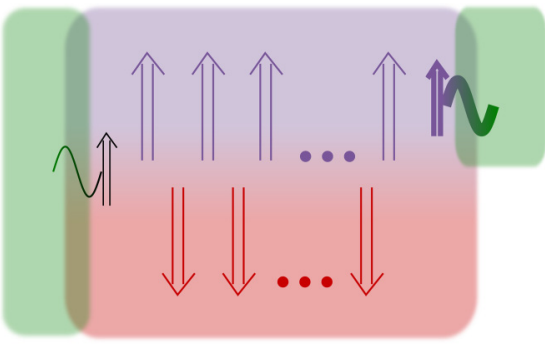

(a)

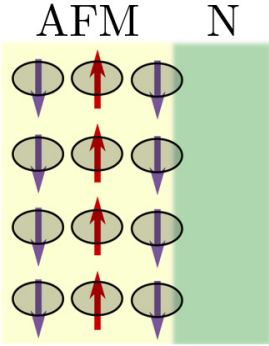

(b)
FIG. 2. (a) An external excitation bath (shaded green) interacts weakly with the AFM squeezed magnon if coupled via its unit net spin (left) but strongly if exposed to only one of the sublattices (right). (b) Schematic depiction of a metal (N) coupled to an AFM via a fully uncompensated interface.

amount dictated by the degree of squeezing, parametrized by the non-negative squeeze parameter $r$. Similarly, a spin-up AFM (squeezed) magnon is composed of a superposition of states with $n+1$ spin-up and $n$ spin-down sublattice magnons [Figs. 1(b) and 1(c)]. Thus, despite its unit net spin, it carries enormous spins on each sublattice, which allows it to couple strongly with other excitations via a sublattice-spinmediated interaction (Fig. 2). Owing to a perfect correlation between the two sublattice-magnon numbers, AFM squeezed magnons are shown to embody entanglement quantified by von Neumann entropy [1,39] increasing monotonically with $r$ (Fig. 3). The degree of squeezing and entanglement embodied by these eigenmodes is significantly larger than that in hitherto achieved nonequilibrium states. We also comment on existing experiments $[40,41]$ where this squeezing-mediated coupling enhancement (Fig. 2) has been observed and strategies for exploiting the entanglement contained in antiferromagnetic magnons. While the squeezed states of light are generated via external drives and are nonequilibrium states [1], the

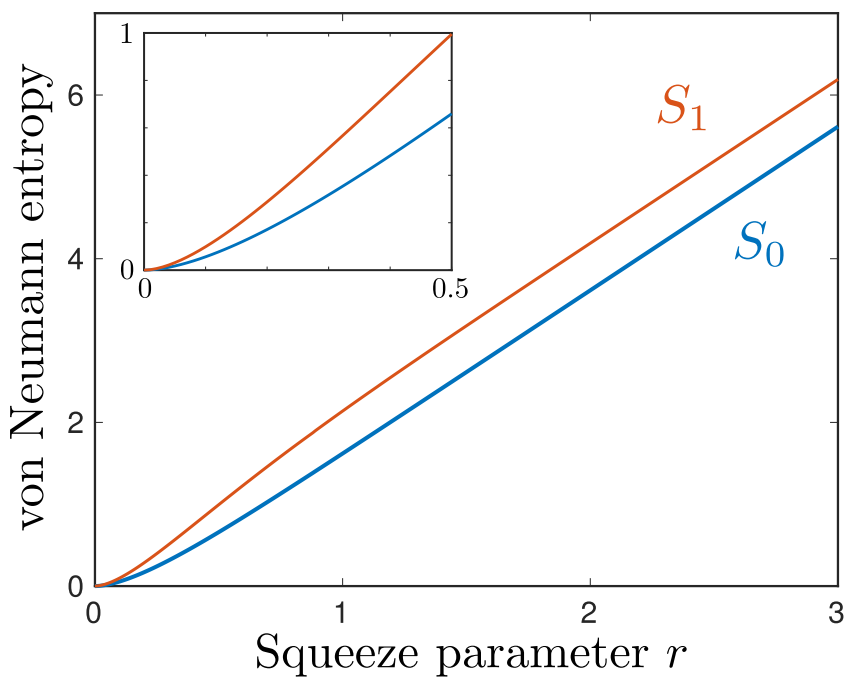

FIG. 3. Entanglement between the two constituent sublattice magnons quantified via von Neumann entropy for the squeezed vacuum $\left(S_{0}\right)$ and magnon $\left(S_{1}\right)$ eigenmodes. The inset shows a zoom of the small- $r$ range. 
antiferromagnetic squeezed magnons are eigenmodes of the system with their squeezing being equilibrium in nature and resulting from energy minimization.

\section{AFM EIGENMODES AS SQUEEZED FOCK STATES}

We consider a Néel ordered ansatz with sublattice A and $\mathrm{B}$ spins pointing along $\hat{z}$ and $-\hat{z}$, respectively. The antiferromagnetic Hamiltonian may then be expressed in terms of the corresponding sublattice-magnon ladder operators $\tilde{a}_{k}, \tilde{b}_{k}$ as $[20,42]$

$$
\tilde{H}=\sum_{k} A_{k}\left(\tilde{a}_{k}^{\dagger} \tilde{a}_{k}+\tilde{b}_{k}^{\dagger} \tilde{b}_{k}\right)+C_{k}\left(\tilde{a}_{k} \tilde{b}_{-k}+\tilde{a}_{k}^{\dagger} \tilde{b}_{-k}^{\dagger}\right),
$$

where we assume inversion symmetry and disregard applied magnetic fields for simplicity. Consistent with the assumed Néel order, sublattice B (A) magnons represented by $\tilde{b}_{\boldsymbol{k}}\left(\tilde{a}_{\boldsymbol{k}}\right)$ are spin up (down). In addition to the general considerations captured by Eq. (1), we will obtain specific results for a uniaxial, easy-axis AFM described by

$$
\begin{aligned}
\tilde{H}_{\mathrm{uni}}= & \frac{J}{\hbar^{2}} \sum_{i, \boldsymbol{\delta}} \tilde{\boldsymbol{S}}_{\mathrm{A}}\left(\boldsymbol{r}_{i}\right) \cdot \tilde{\boldsymbol{S}}_{\mathrm{B}}\left(\boldsymbol{r}_{i}+\boldsymbol{\delta}\right) \\
& -\frac{K}{\hbar^{2}} \sum_{i}\left[\tilde{S}_{\mathrm{A} z}\left(\boldsymbol{r}_{i}\right)\right]^{2}-\frac{K}{\hbar^{2}} \sum_{j}\left[\tilde{S}_{\mathrm{B} z}\left(\boldsymbol{r}_{j}\right)\right]^{2} .
\end{aligned}
$$

Here, the positive parameters $J$ and $K$ account for intersublattice antiferromagnetic exchange and easy-axis anisotropy, respectively. $\tilde{\boldsymbol{S}}_{\mathrm{A}, \mathrm{B}}$ represent the respective spin operators, $\boldsymbol{r}_{i}\left(\boldsymbol{r}_{j}\right)$ runs over the sublattice A (B), and $\boldsymbol{\delta}$ are vectors to the nearest neighbors. Executing Holstein-Primakoff transformations [43] and switching to Fourier space, Eq. (2) reduces to Eq. (1) apart from a constant-energy offset [20,44], with $A_{k}=$ $J S z+2 K S$ and $C_{k}=J S z \gamma_{k}$. Here, $S$ is the spin on each site, $z$ is the coordination number, and $\gamma_{\boldsymbol{k}} \equiv(1 / z) \sum_{\delta} \exp (i \boldsymbol{k} \cdot \boldsymbol{\delta})$.

The Hamiltonian [Eq. (1)] is diagonalized to $\tilde{H}=$ $\sum_{k} \epsilon_{k}\left(\tilde{\alpha}_{k}^{\dagger} \tilde{\alpha}_{k}+\tilde{\beta}_{k}^{\dagger} \tilde{\beta}_{k}\right)$ via a Bogoliubov transformation [43] described by [45]:

$$
\begin{array}{cc}
\tilde{\alpha}_{k}=u_{k} \tilde{a}_{k}+v_{k} \tilde{b}_{-k}^{\dagger}, & \tilde{\beta}_{k}=u_{k} \tilde{b}_{k}+v_{k} \tilde{a}_{-k}^{\dagger}, \\
u_{k}=\sqrt{\frac{A_{k}+\epsilon_{k}}{2 \epsilon_{k}}}, & v_{k}=\sqrt{\frac{A_{k}-\epsilon_{k}}{2 \epsilon_{k}}},
\end{array}
$$

where $\epsilon_{k}=\sqrt{A_{k}^{2}-C_{k}^{2}} \cdot \tilde{\alpha}_{k}$ and $\tilde{\beta}_{k}$ represent the spin-down and -up eigenmodes of the AFM, which are subsequently called squeezed magnons. Denoting the resulting antiferromagnetic vacuum or ground-state wave function by $|G\rangle_{\mathrm{sq}}$, we have $\tilde{\alpha}_{k}|G\rangle_{\mathrm{sq}}=\tilde{\beta}_{k}|G\rangle_{\mathrm{sq}}=0$ for all $\boldsymbol{k}$.

Let us first consider the spatially uniform modes, i.e., $\boldsymbol{k}=\mathbf{0}$. We denote states in the corresponding reduced subspaces via $\left|N_{b_{0}}, N_{a_{0}}\right\rangle_{\text {sub }}$ and $\left|N_{\beta_{0}}, N_{\alpha_{0}}\right\rangle_{\text {sq }}$, where $N_{b_{0}}$ denotes the number of spin-up sublattice magnons and so on. Within the reduced subspaces, the Néel ordered state is thus denoted by $|0,0\rangle_{\text {sub }}$, while the antiferromagnetic ground state obtained above is represented by $|0,0\rangle_{\mathrm{sq}}$. We define the relevant twomode squeeze operator [1]: $\tilde{S}_{2}\left(r_{0}\right) \equiv \exp \left(r_{0} \tilde{a}_{0} \tilde{b}_{0}-r_{0} \tilde{a}_{0}^{\dagger} \tilde{b}_{0}^{\dagger}\right)$, with the non-negative squeeze parameter $r_{0}$ given via $u_{0} \equiv$ $\cosh r_{0}$ and $v_{0} \equiv \sinh r_{0}$ [Eq. (4)] [46]. Employing the identities [1,19]

$$
\tilde{\alpha}_{0}=\tilde{S}_{2}\left(r_{0}\right) \tilde{a}_{0} \tilde{S}_{2}^{-1}\left(r_{0}\right), \quad \tilde{\beta}_{0}=\tilde{S}_{2}\left(r_{0}\right) \tilde{b}_{0} \tilde{S}_{2}^{-1}\left(r_{0}\right),
$$

where $\tilde{\alpha}_{0}$ and $\tilde{\beta}_{0}$ are given by Eq. (3), in the condition $\tilde{\alpha}_{0}|0,0\rangle_{\mathrm{sq}}=\tilde{\beta}_{0}|0,0\rangle_{\mathrm{sq}}=0$, we obtain

$$
|0,0\rangle_{\mathrm{sq}}=\tilde{S}_{2}\left(r_{0}\right)|0,0\rangle_{\mathrm{sub}} \text {. }
$$

Thus, the uniform modes' antiferromagnetic ground state is a two-mode squeezed vacuum of sublattice magnons. The complementary demonstration of quadrature squeezing is detailed in Appendix A. Working along the same lines as above, it is straightforward to show that $|m, n\rangle_{\mathrm{sq}}=$ $\tilde{S}_{2}\left(r_{0}\right)|m, n\rangle_{\text {sub }}$, thereby demonstrating the antiferromagnetic eigenmodes are two-mode squeezed sublattice-magnon Fock states. Therefore, the eigenmodes are henceforth called "squeezed magnons."

Based on the analysis above, it becomes evident that the antiferromagnetic ground state is obtained by pairwise, twomode squeezing of the Néel ordered state:

$$
|G\rangle_{\mathrm{sq}}=\left[\prod_{k} \tilde{S}_{2}\left(r_{\boldsymbol{k}}\right)\right]|\mathrm{Néel}\rangle_{\mathrm{sub}},
$$

where $\tilde{S}_{2}\left(r_{k}\right) \equiv \exp \left(r_{k} \tilde{a}_{k} \tilde{b}_{-k}-r_{k} \tilde{a}_{k}^{\dagger} \tilde{b}_{-k}^{\dagger}\right)$, with the squeeze parameters $r_{k}$ given via $u_{k}=u_{-k} \equiv \cosh r_{k}$. The $\tilde{\alpha}_{k}$ eigenmode is thus a two-mode $\left(\tilde{a}_{k}\right.$ and $\left.\tilde{b}_{-k}\right)$ squeezed magnon [Eq. (3)]. Similarly, the $\tilde{\beta}_{\boldsymbol{k}}$ eigenmode is also a two-mode squeezed magnon formed by $\tilde{b}_{k}$ and $\tilde{a}_{-k}$ modes [Eq. (3)]. Due to this mathematical equivalence, it suffices to analyze the spatially uniform eigenmodes, which is what we focus on in the following.

\section{SPATIALLY UNIFORM EIGENMODES}

For ease of notation, we denote the wave functions for a spatially uniform squeezed vacuum by $|0\rangle_{\mathrm{sq}}$ and spin-up squeezed magnon by $|\uparrow\rangle_{\mathrm{sq}}$, while the corresponding squeeze parameter is denoted by $r$. Considering a uniaxial AFM [Eq. (2)], we obtain $\cosh r \approx(1 / 2)(J z / K)^{1 / 4}$ [Eq. (4)], which translates to $r \approx 3$ for a typical ratio of $J / K \sim 10^{4}$. To get a feel for numbers, the most squeezed vacuum state of light generated so far corresponds to a squeeze parameter of about $1.7[2,47]$. Furthermore, in the limit $K \rightarrow 0$, the squeeze parameter is found to diverge. This feature is general and a direct consequence [Eq. (4)] of the Goldstone theorem, according to which $\epsilon_{0} \rightarrow 0$ in the limit of isotropy.

Employing the relation $\tilde{\alpha}_{0}|0\rangle_{\mathrm{sq}}=\left(\cosh r \tilde{a}_{0}+\right.$ $\left.\sinh r \tilde{b}_{0}^{\dagger}\right)|0\rangle_{\mathrm{sq}}=0$, the squeezed vacuum is obtained in terms of the uniform sublattice-magnons subspace [1]:

$$
|0\rangle_{\mathrm{sq}}=\sum_{n=0}^{\infty} \frac{(-\tanh r)^{n}}{\cosh r}|n, n\rangle_{\mathrm{sub}} \equiv \sum_{n} P_{n}|n, n\rangle_{\mathrm{sub}} .
$$

The ensuing wave function is schematically depicted in Fig. 1(a), and the distribution over constituent states is plotted in Fig. 1(c). With an increasing $r$, the number of states that contribute substantially to the superposition increases monotonically. This presence of sublattice magnons in the ground state constitutes quantum fluctuations.

A similar representation for the spin-up squeezed magnon is obtained via $|\uparrow\rangle_{\mathrm{sq}}=\tilde{\beta}_{0}^{\dagger}|0\rangle_{\mathrm{sq}}=\left(\cosh r \tilde{b}_{0}^{\dagger}+\sinh r \tilde{a}_{0}\right)|0\rangle_{\mathrm{sq}}$ 
and Eq. (8):

$$
\begin{aligned}
|\uparrow\rangle_{\mathrm{sq}} & =\sum_{n=0}^{\infty} \frac{\sqrt{n+1}(-\tanh r)^{n}}{\cosh ^{2} r}|n+1, n\rangle_{\mathrm{sub}} \\
& \equiv \sum_{n} Q_{n}|n+1, n\rangle_{\mathrm{sub}} .
\end{aligned}
$$

A schematic depiction and the distribution over constituent states are shown in Figs. 1(b) and 1(c). In stark contrast to the squeezed vacuum, where the contribution from states decreases monotonically with $n$, the highest contribution to the superposition here comes from $n \approx \sinh ^{2} r$. No such peak exists for weak squeezing when $\sinh r<1$. The average number of spin-up magnons comprising a squeezed magnon is evaluated as $\cosh ^{2} r+\sinh ^{2} r$. Thus, a typical AFM squeezed magnon, corresponding to $r \approx 3$ estimated above, is composed of around 200 spin-up magnons on one sublattice and nearly the same number of spin-down magnons on the other. It is thus an enormous excitation, despite its unit net spin.

\section{ENHANCED INTERACTION}

This enormous nature of the AFM squeezed magnon reveals an approach to exploit it. When it couples to excitations, such as itinerant electrons or phonons, via its net spin, the interaction strength is proportional to the relatively small unit spin. On the other hand, if an interaction is mediated via the sublattice spin, it will be greatly enhanced (by a factor $\sim \cosh ^{2} r \approx 100$ for $r \approx 3$ ) on account of its large sublattice spin content [Fig. 2(a)]. Such a situation arises, for example, when an AFM is exposed to a metal via an uncompensated interface [Fig. 2(b)] [26,48-50]. This effect provides a physical picture for the theoretically encountered enhancement in spin pumping current from AFM into an adjacent conductor coupled asymmetrically to the two sublattices [49]. The same mechanism has also been exploited in predicting an enhanced magnon-mediated superconductivity in a conductor bearing an uncompensated interface with an AFM [51]. Rigorous derivations of electron-magnon and magnon-magnon couplings presented, respectively, in Appendixes B and C demonstrate an enhancement of the interactions consistent with the intuition above, reinforcing the generality of this phenomenon.

\section{ENTANGLEMENT}

In a two-mode squeezed vacuum, the participating modes are entangled with the degree of entanglement quantified by the von Neumann entropy $[1,39] S_{0}$ :

$$
\begin{aligned}
S_{0} & =-\sum_{n}\left|P_{n}\right|^{2} \ln \left(\left|P_{n}\right|^{2}\right) \\
& =2 \ln (\cosh r)-2\left(\sinh ^{2} r\right) \ln (\tanh r) .
\end{aligned}
$$

Such two-mode squeezed vacuum states of light have been exploited for obtaining useful entanglement [7]. This high von Neumann entropy content of our squeezed-magnon vacuum can be exploited, for example, in entangling two qubits [52] coupled respectively to sublattices A and B. Furthermore, the squeezed magnons themselves embody strong entanglement, quantified by an even larger von Neumann entropy $S_{1}=$ $-\sum_{n}\left|Q_{n}\right|^{2} \ln \left(\left|Q_{n}\right|^{2}\right)$ (Fig. 3), which may be transferred to external excitations. This can be achieved by coupling the systems to be entangled with the opposite sublattices [53-57] via uncompensated interfaces [Fig. 2(b)], for example, as has been detailed further in Appendix D. In comparison, von Neumann entropy [58] of about 1 has been measured in cold-atom systems [59]. This high von Neumann entropy content and the large number of entangled spins $\left(\sim \cosh ^{2} r\right)$ that comprise the AFM squeezed magnon make it an entangled excitation complementary to the "massively entangled" excitations hosted by some quantum spin liquids [36-38].

\section{QUANTUM FLUCTUATIONS IN "CLASSICAL" EXPERIMENTS}

The interaction enhancement effect [Fig. 2(a)] is rooted in high magnon squeezing and the underlying quantum superposition of a large number of states [Eq. (9)]. It is a direct consequence of the strong quantum fluctuations in the antiferromagnetic ground state, which hosts this excitation, and is thus a quantum fluctuation effect itself. Nevertheless, this coupling enhancement is observed as an increased magnetic damping around the compensation temperature in a compensated ferrimagnet [40], which mimics an AFM [20,60]. Recently, this enhancement was observed and exploited in a compensated ferrimagnet for an ultrastrong magnon-magnon coupling, resulting in hybridization between the two enormous spin-up and -down squeezed magnons [41]. These classical experiments at high temperatures may thus be considered an observation of the antiferromagnetic quantum fluctuations. As detailed in Appendix $\mathrm{C}$, this large squeezing-mediated enhancement $(\sim \sqrt{J / K}$ for our uniaxial AFM), suggested recently in the context of light-matter interaction [61,62], is reproduced by the classical theory of spin dynamics $[41,60]$, where it is termed "exchange enhancement." This is understandable since the classical dynamics is captured by the quantum system being in a coherent state $[49,63,64]$, which fully accounts for the average effect of these quantum fluctuations.

\section{GENERALIZATIONS}

The description in terms of squeezed Fock states developed herein is a mathematical consequence of the Bogoliubov transformation and goes beyond AFMs. It should allow a similar physical picture and subsequent exploitation of quantum effects in other systems such as cold atoms [65-67]. Here, we have disregarded the relatively weak spin-nonconserving interactions. Inclusion of those necessitates a four-dimensional Bogoliubov transform [20], thereby precluding the simple two-mode squeezed Fock state description employed here. Similar complications also arise when considering AFMs lacking inversion symmetry. Nevertheless, an analogous general picture can be developed.

\section{CONCLUSION}

We have developed a description and physical picture of an antiferromagnetic ground state and excitations based on the concept of two-mode squeezed Fock states. Capitalizing 
on the tremendous progress in quantum optics, these fresh insights pave the way for exploiting the quantum properties of antiferromagnetic squeezed magnons towards potentially room temperature quantum devices.

\section{ACKNOWLEDGMENTS}

A.K. thanks S. Takei, L. Liensberger, M. Weiler, and H. Huebl for valuable discussions. We acknowledge financial support from the Research Council of Norway through its Centers of Excellence funding scheme, Project No. 262633, "QuSpin," and the DFG through SFB 767. A.S. also acknowledges support from the Research Council of Norway, Grant No. 250985, "Fundamentals of Low-dissipative Topological Matter."

\section{APPENDIX A: DEMONSTRATION OF QUADRATURE SQUEEZING}

In this Appendix, we clarify the squeezed nature of the antiferromagnetic ground state by evaluating the quantum fluctuations in the appropriate quadratures. This approach is complementary to the more general discussion in terms of the two-mode squeeze operator [1] presented in the main text. Once again, we focus on the uniform modes, i.e., $\boldsymbol{k}=\mathbf{0}$, recognizing that the corresponding results for $\boldsymbol{k} \neq \mathbf{0}$ follow in a similar fashion. We first demonstrate the quadrature squeezing following the standard approach within quantum optics [1] and physically interpret the quadratures later.

For the two-mode squeezing of $\tilde{a}_{0}$ and $\tilde{b}_{0}$ that is operational here, the relevant quadratures are formed via a combination of both modes' ladder operators [1]:

$$
\begin{aligned}
& \tilde{X}_{1} \equiv \frac{1}{\sqrt{8}}\left(\tilde{a}_{0}+\tilde{a}_{0}^{\dagger}+\tilde{b}_{0}+\tilde{b}_{0}^{\dagger}\right), \\
& \tilde{X}_{2} \equiv \frac{1}{i \sqrt{8}}\left(\tilde{a}_{0}-\tilde{a}_{0}^{\dagger}+\tilde{b}_{0}-\tilde{b}_{0}^{\dagger}\right) .
\end{aligned}
$$

Employing the bosonic commutation relations of the ladder operators, we obtain $\left[\tilde{X}_{1}, \tilde{X}_{2}\right]=i / 2$, demonstrating that the chosen quadratures of Eqs. (A1) and (A2) represent two noncommuting observables. Denoting the reduced subspace of the uniform modes within the Néel ordered state by $|0\rangle_{\text {sub }}$, the quantum fluctuations in the two quadratures are evaluated as

$$
\begin{gathered}
\left\langle\left. 0\right|_{\text {sub }}\left(\delta \tilde{X}_{1}\right)^{2} \mid 0\right\rangle_{\text {sub }} \equiv\left\langle\left. 0\right|_{\text {sub }}\left(\tilde{X}_{1}-\left\langle\tilde{X}_{1}\right\rangle\right)^{2} \mid 0\right\rangle_{\text {sub }}=\frac{1}{4}, \\
\left\langle\left. 0\right|_{\text {sub }}\left(\delta \tilde{X}_{2}\right)^{2} \mid 0\right\rangle_{\text {sub }}=\frac{1}{4} .
\end{gathered}
$$

Therefore, the two quadratures host equal quantum noise in the Néel ordered state, that is, $\left\langle\left. 0\right|_{\text {sub }}\left(\delta \tilde{X}_{1}\right)^{2} \mid 0\right\rangle_{\text {sub }}=$ $\left\langle\left. 0\right|_{\text {sub }}\left(\delta \tilde{X}_{2}\right)^{2} \mid 0\right\rangle_{\text {sub }}$.

We now consider fluctuations in the antiferromagnetic ground state with the uniform modes' reduced subspace denoted by $|0\rangle_{\mathrm{sq}}$, as in the main text. Employing the Bogoliubov transformation relations $\tilde{a}_{0}=\cosh r \tilde{\alpha}_{0}-\sinh r \tilde{\beta}_{0}^{\dagger}$ and $\tilde{b}_{0}=$ $\cosh r \tilde{\beta}_{0}-\sinh r \tilde{\alpha}_{0}^{\dagger}$, the two quadratures can be expressed as

$$
\begin{aligned}
& \tilde{X}_{1}=\frac{\cosh r-\sinh r}{\sqrt{8}}\left(\tilde{\alpha}_{0}+\tilde{\alpha}_{0}^{\dagger}+\tilde{\beta}_{0}+\tilde{\beta}_{0}^{\dagger}\right), \\
& \tilde{X}_{2}=\frac{\cosh r+\sinh r}{i \sqrt{8}}\left(\tilde{\alpha}_{0}-\tilde{\alpha}_{0}^{\dagger}+\tilde{\beta}_{0}-\tilde{\beta}_{0}^{\dagger}\right) .
\end{aligned}
$$

Employing the quadrature expressions thus obtained, quantum fluctuations in the antiferromagnetic ground state are conveniently evaluated as

$$
\begin{aligned}
\left\langle\left. 0\right|_{\mathrm{sq}}\left(\delta \tilde{X}_{1}\right)^{2} \mid 0\right\rangle_{\mathrm{sq}} & =\frac{(\cosh r-\sinh r)^{2}}{4}=\frac{e^{-2 r}}{4}, \\
\left\langle\left. 0\right|_{\mathrm{sq}}\left(\delta \tilde{X}_{2}\right)^{2} \mid 0\right\rangle_{\mathrm{sq}} & =\frac{(\cosh r+\sinh r)^{2}}{4}=\frac{e^{2 r}}{4},
\end{aligned}
$$

thereby demonstrating the quadrature squeezing [1] of the antiferromagnetic ground state, that is, $\left\langle\left. 0\right|_{\mathrm{sq}}\left(\delta \tilde{X}_{1}\right)^{2} \mid 0\right\rangle_{\mathrm{sq}}<$ $\left\langle\left. 0\right|_{\mathrm{sq}}\left(\delta \tilde{X}_{2}\right)^{2} \mid 0\right\rangle_{\mathrm{sq}}$.

We now relate the two quadratures [Eqs. (A1) and (A2)] with physical observables of the antiferromagnet (AFM). Employing Fourier relations of the kind

$$
\tilde{a}_{k}=\frac{1}{\sqrt{N}} \sum_{i} \tilde{a}_{i} e^{i \boldsymbol{k} \cdot \boldsymbol{r}_{i}},
$$

in conjunction with the linearized Holstein-Primakoff transformations for the AFM $[42,44]$,

$$
\begin{gathered}
\tilde{S}_{A+}\left(\boldsymbol{r}_{i}\right)=\tilde{S}_{A x}\left(\boldsymbol{r}_{i}\right)+i \tilde{S}_{A y}\left(\boldsymbol{r}_{i}\right)=\hbar \sqrt{2 S} \tilde{a}_{i}, \\
\tilde{S}_{B+}\left(\boldsymbol{r}_{j}\right)=\tilde{S}_{B x}\left(\boldsymbol{r}_{j}\right)+i \tilde{S}_{B y}\left(\boldsymbol{r}_{j}\right)=\hbar \sqrt{2 S} \tilde{b}_{j}^{\dagger},
\end{gathered}
$$

we obtain

$$
\begin{aligned}
& \tilde{X}_{1}=\frac{1}{2 \hbar \sqrt{N S}}\left(\tilde{S}_{A x}+\tilde{S}_{B x}\right), \\
& \tilde{X}_{2}=\frac{1}{2 \hbar \sqrt{N S}}\left(\tilde{S}_{A y}-\tilde{S}_{B y}\right) .
\end{aligned}
$$

Here, $N$ is the total number of sites on each sublattice, $S$ is the spin at each site as defined in the main text, and $\tilde{S}_{A x} \equiv$ $\sum_{i} \tilde{S}_{A x}\left(\boldsymbol{r}_{i}\right)$ is the $x$ component of the total spin on sublattice A and so on. Thus, the two quadratures are related to the $x$ and $y$ components of the total spin and the Néel order, respectively.

In the qualitatively distinct case of single-mode squeezing manifested by the uniform mode in an anisotropic ferromagnet [19], the two quadratures are simply the $x$ and $y$ components of the total spin, providing a geometrical "ellipticity" interpretation to the squeezing effect [68]. In contrast, the situation is less intuitive for the case of two-mode squeezing as the ellipticity of quantum fluctuations exists in a more abstract space. In the present case, this space is defined by the transverse orthogonal components of the total spin and the Néel order associated with the AFM [Eqs. (A12) and (A13)].

\section{APPENDIX B: ELECTRON-MAGNON COUPLING}

Heterostructures in which a magnetic insulator layer interfaces with another material hosting conduction electrons have emerged as basic building blocks in a wide range of spintronic concepts and devices. The interfacial exchange-mediated coupling between the magnons in the former and the electrons in the latter have enabled magnon-based information processing schemes, magnon-mediated condensation phenomena, and so on. Thus, an ability to engineer and amplify the electronmagnon coupling is expected to have a strong and broad impact. In this section, we discuss the electron-magnon coupling in an AFM/normal-metal (N) bilayer with the goal of highlighting this tunability and amplification of electron-magnon 
coupling by exploiting the squeezing effect, as discussed in the main text. A thorough analysis of this system along with spin transport effects has been provided elsewhere [49]. We here focus on highlighting the amplification effect for an uncompensated AFM with respect to other related systems, providing mathematical expressions complementary to the intuitive physical picture discussed in the main text.

The AFM and $\mathrm{N}$ layers are assumed to interact via interfacial exchange, resulting in the following contribution to the Hamiltonian [49] within a continuum model:

$$
\tilde{H}_{\mathrm{int}}=-\frac{1}{\hbar^{2}} \int_{\mathcal{A}} d^{2} \rho \sum_{\mathrm{G}=\mathrm{A}, \mathrm{B}} \mathcal{J}_{\mathrm{iG}} \tilde{S}_{\mathrm{G}}(\boldsymbol{\rho}) \cdot \tilde{S}_{\mathrm{N}}(\boldsymbol{\rho}),
$$

where $\mathcal{A}$ is the interfacial area, $\boldsymbol{\rho}$ is the two-dimensional position vector in the interfacial plane, $\tilde{S}_{\mathrm{N}}$ is the conduction electron spin density operator in N, $\tilde{S}_{\mathrm{G}}$ is the spin density operator in the magnet for sublattice $\mathrm{G}$, and $\mathcal{J}_{\mathrm{iG}}$ parametrizes the exchange interaction between the two spin densities, allowing it to be sublattice asymmetric. In terms of the ladder operators for the conduction electrons and magnons, the Hamiltonian above takes the form

$$
\tilde{H}_{\mathrm{int}}=\hbar \sum_{\boldsymbol{q}_{1}, \boldsymbol{q}_{2}, \boldsymbol{k}} \tilde{c}_{\boldsymbol{q}_{1}+}^{\dagger} \tilde{c}_{\boldsymbol{q}_{2}-}\left(W_{\boldsymbol{q}_{1} \boldsymbol{q}_{2} \boldsymbol{k}}^{\mathrm{A}} \tilde{a}_{\boldsymbol{k}}+W_{\boldsymbol{q}_{1} \boldsymbol{q}_{2}}^{\mathrm{B}} \tilde{b}_{\boldsymbol{k}}^{\dagger}\right)+\text { H.c. }
$$

where $\tilde{c}_{q+}$ denotes the annihilation operator for the $\mathrm{N}$ conduction electron with wave vector $\boldsymbol{q}$ and spin $+\hbar / 2$ along the $z$ direction and so on, $\tilde{a}_{k}$ and $\tilde{b}_{k}$ are the annihilation operators for the sublattice magnons as discussed in the main text, and $W_{q_{1} q_{2} k}^{\mathrm{A}}$ is the appropriate amplitude given by the overlap integral between the participating excitation wave functions [49]. With the aim of focusing on the key ingredient in enhancing the coupling, we henceforth consider the relevant and simplified part of the Hamiltonian [enclosed by parentheses in Eq. (B2)] describing electron-magnon coupling:

$$
\tilde{P}=W^{\mathrm{A}} \tilde{a}_{0}+W^{\mathrm{B}} \tilde{b}_{\mathbf{0}}^{\dagger},
$$

where we have again specialized the expression to uniform $(\boldsymbol{k}=\mathbf{0})$ modes for simplicity and $W^{\mathrm{A}, \mathrm{B}} \propto \mathcal{J}_{\mathrm{iA}, \mathrm{BB}}$ capture the sublattice-asymmetry in the interfacial coupling.

For comparison, we first consider the case of a singlesublattice isotropic ferromagnet [19] for which the interaction is described simply by $\tilde{P}=W \tilde{a}_{0}$, with $\tilde{a}_{0}$ representing the normal magnon mode. The transition rate $\Gamma$ for the electronmagnon scattering process is thus simply determined by $W$, i.e., $\Gamma \propto|W|^{2}$. For the case of AFMs, in contrast, Eq. (B3) becomes

$$
\begin{aligned}
\tilde{P}= & \left(\cosh r W^{\mathrm{A}}-\sinh r W^{\mathrm{B}}\right) \tilde{\alpha}_{0} \\
& +\left(\cosh r W^{\mathrm{B}}-\sinh r W^{\mathrm{A}}\right) \tilde{\beta}_{0}^{\dagger}
\end{aligned}
$$

in terms of the normal magnon modes. Now considering $W^{\mathrm{A}}=W^{\mathrm{B}} \equiv W$ for a compensated interface, in which the two sublattices couple equally to the $\mathrm{N}$ electrons, we obtain

$$
\tilde{P}=W(\cosh r-\sinh r) \tilde{\alpha}_{0}+W(\cosh r-\sinh r) \tilde{\beta}_{0}^{\dagger},
$$

where we see that the transition rate is reduced: $\Gamma \propto$ $(\cosh r-\sinh r)^{2}|W|^{2} \approx|W|^{2} /\left(4 \cosh ^{2} r\right)$, accounting for the large squeezing such that $\cosh r \gg 1$. The electronmagnon coupling for this case is thus suppressed compared to that for ferromagnetic magnons considered above. Arriving at the crux of this Appendix, as discussed in the main text, when the coupling is mediated by the sublattice spin of the magnon via an uncompensated interface $\left(W^{\mathrm{A}}=W, W^{\mathrm{B}}=0\right)$, we obtain

$$
\tilde{P}=W \cosh r \tilde{\alpha}_{0}-W \sinh r \tilde{\beta}_{0}^{\dagger} .
$$

The transition rates for the electron-magnon scattering processes are thus given by $\Gamma \propto \cosh ^{2} r|W|^{2}$ for the $\tilde{\alpha}_{0}$ mode and $\Gamma \propto \sinh ^{2} r|W|^{2} \approx \cosh ^{2} r|W|^{2}$ for the $\tilde{\beta}_{0}$ mode. Thus, we find a squeezing-mediated enhancement in the electronmagnon coupling for the case of sublattice spin-mediated interaction. Furthermore, this is consistent with the simple picture discussed in the main text, and the interaction enhancement factor is related to the sublattice spin associated with a single eigenexcitation: the antiferromagnetic squeezed magnon.

\section{APPENDIX C: MAGNON-MAGNON COUPLING}

In this Appendix, we investigate coupling between the two opposite-spin antiferromagnetic eigenmodes caused by a spinnonconserving interaction [20]. In particular, we demonstrate that a sublattice spin-mediated magnon-magnon coupling is amplified via the squeezing effect, in agreement with the general picture discussed in the main text. This also provides a derivation, within the quantum picture, for the recently observed "exchange-enhanced" ultrastrong magnon-magnon coupling in a compensated ferrimagnet [41] without accounting for all the experimental complexities therein.

In the main text, we considered only interactions that conserve the $z$-projected spin of the AFM. The diagonalized Hamiltonian therefore assumes the form

$$
\tilde{H}=\sum_{k} \epsilon_{k}\left(\tilde{\alpha}_{k}^{\dagger} \tilde{\alpha}_{k}+\tilde{\beta}_{k}^{\dagger} \tilde{\beta}_{k}\right),
$$

with the two opposite-spin squeezed magnons as degenerate excitations of the system, in the absence of an applied field. However, breaking the spin conservation [69] in the system allows us to couple these opposite-spin excitations, resulting in a lifting of degeneracy and the concomitant hybridization [20]. As discussed in the main text, accounting for such spinnonconserving terms necessitates a four-dimensional Bogoliubov transform for an exact diagonalization of the Hamiltonian [20]. Here, we circumvent this mathematical complexity by describing the mode coupling in a perturbative manner, treating Eq. (C1) and squeezed magnons as our unperturbed Hamiltonian and eigenexcitations, respectively. This allows us to obtain an analytic expression for the coupling rate while appreciating and justifying the typical approximations employed in such descriptions [1].

For concreteness, we consider the following spinnonconserving and sublattice spin-mediated contribution to the Hamiltonian that may stem from the magnetocrystalline anisotropy [41]:

$$
\tilde{H}_{\text {coup }}=\frac{K_{a}}{\hbar^{2}} \sum_{i}\left[\tilde{S}_{A x}\left(\boldsymbol{r}_{i}\right)\right]^{2}+\frac{K_{a}}{\hbar^{2}} \sum_{j}\left[\tilde{S}_{B x}\left(\boldsymbol{r}_{j}\right)\right]^{2},
$$

where $K_{a}$ parametrizes this axial-symmetry-breaking anisotropy and the rest of the notation was already introduced in the main text. Employing the Holstein-Primakoff 
transformation and switching to Fourier space, the coupling Hamiltonian above is brought to the following form:

$$
\tilde{H}_{\text {coup }}=\frac{K_{a} S}{2} \sum_{k} \tilde{a}_{k}^{\dagger} \tilde{a}_{-k}^{\dagger}+\tilde{b}_{k}^{\dagger} \tilde{b}_{-k}^{\dagger}+\tilde{a}_{k} \tilde{a}_{-k}+\tilde{b}_{k} \tilde{b}_{-k}
$$

In writing Eq. (C3), we have neglected terms of the type $\sim \tilde{a}_{k}^{\dagger} \tilde{a}_{k}$ since they can be absorbed into Eq. (C1), leading to a small renormalization of the unperturbed squeezed-magnon energies. We again focus on the uniform modes $(\boldsymbol{k}=\mathbf{0})$ as they are also the ones observed experimentally [41]:

$$
\tilde{H}_{\text {coup }}(\boldsymbol{k}=\mathbf{0})=\frac{K_{a} S}{2}\left(\tilde{a}_{0}^{2}+\tilde{b}_{0}^{2}+\text { H.c. }\right) \text {. }
$$

Employing the Bogoliubov transformation relations $\tilde{a}_{0}=$ $\cosh r \tilde{\alpha}_{0}-\sinh r \tilde{\beta}_{0}^{\dagger}$ and $\tilde{b}_{0}=\cosh r \tilde{\beta}_{0}-\sinh r \tilde{\alpha}_{0}^{\dagger}$, the coupling Hamiltonian may be expressed in terms of the unperturbed eigenexcitations:

$$
\begin{aligned}
& \tilde{H}_{\text {coup }}(\boldsymbol{k}=\mathbf{0})=-\cosh r \sinh r 2 K_{a} S\left(\tilde{\alpha}_{0} \tilde{\beta}_{0}^{\dagger}+\tilde{\alpha}_{0}^{\dagger} \tilde{\beta}_{0}\right) \\
&+\frac{K_{a} S\left(\cosh ^{2} r+\sinh ^{2} r\right)}{2}\left(\tilde{\alpha}_{0}^{2}+\tilde{\beta}_{0}^{2}+\text { H.c. }\right) \\
& \approx-\cosh r \sinh r 2 K_{a} S\left(\tilde{\alpha}_{0} \tilde{\beta}_{0}^{\dagger}+\tilde{\alpha}_{0}^{\dagger} \tilde{\beta}_{0}\right) .
\end{aligned}
$$

In the last simplification above, we have employed the rotating wave approximation [1] and disregarded terms which merely cause rapid oscillations.

Equation (C6) constitutes the main result of this Appendix, where the coupling rate can be read off as $\cosh r \sinh r 2 K_{a} S$. The squeezing-mediated enhancement in the coupling of $\cosh r \sinh r \approx \cosh ^{2} r \sim \sqrt{J / K}$ is evident and consistent with the intuitive picture presented in the main text. In comparison, if we consider a net spin-mediated magnon-magnon coupling via, for example,

$$
\tilde{H}_{\text {coup }}=\frac{K_{a}}{\hbar^{2}} \sum\left[\tilde{S}_{A x}\left(\boldsymbol{r}_{i}\right)+\tilde{S}_{B x}\left(\boldsymbol{r}_{j}\right)\right]^{2},
$$

an analogous procedure yields a suppressed coupling rate of $K_{a} S /\left(4 \cosh ^{2} r\right)$, in agreement with the electron-magnon coupling considerations discussed above.

Thus, these two instances (electron-magnon and magnonmagnon couplings) of detailed calculations reinforce the generality of the intuitive picture discussed in the main text. This also suggests these coupling properties are intrinsic to the antiferromagnetic squeezed magnons and therefore applicable to a yet wider class of phenomena involving antiferromagnets. We further note that the squeezing-mediated coupling enhancement that we describe here is mathematically analogous to similar nonequilibrium enhancements suggested recently in the context of light-matter interaction [61,62]. Our suggestion for magnets bears advantages such as stronger enhancement, an equilibrium nature of the effect, tunability via temperature

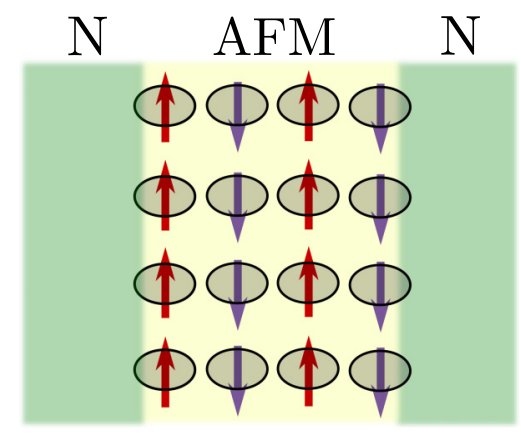

FIG. 4. Schematic depiction of a trilayer heterostructure that allows coupling the two antiferromagnetic sublattices to two different normal metals.

[41], and the recent experimental observation [41] along with the concomitant proof of concept.

\section{APPENDIX D: ACCESSING ENTANGLED SUBSYSTEMS}

The von Neumann entropy is widely employed as a measure to quantify entanglement between two subsystems. Thus, its value depends on how a larger system is partitioned into its entangled constituents. In the case of quantum spin liquids, it is common to draw an imaginary boundary and partition the magnet spatially into inside and outside regions. The entanglement entropy may then be evaluated between these two spatial regions and allows us to determine the entangled and/or topological nature of the ground state as well as excitations. On the other hand, in the case of two-mode squeezed states, the participating modes provide a natural partitioning for entanglement [1]. The participating modes are entangled, which may be exploited for useful protocols [1]. However, to this end, it is crucial to access the two entangled modes separately.

As discussed in the main text, antiferromagnetic squeezed magnons are composed of the two-mode squeezing of the sublattice magnons. Therefore, in order to utilize the squeezing-mediated intrinsic entanglement between the sublattice magnons, it is important to access the sublattice magnons individually. This can be achieved by employing AFMs with two uncompensated interfaces in a trilayer structure as depicted in Fig. 4. Similar heterostructures have also been proposed to host magnon-mediated indirect exciton condensation [56]. The experimental methods and relevant materials for achieving uncompensated interfaces have been discussed elsewhere [50]. Furthermore, the recently discovered layered van der Waals AFMs [57] provide another promising route towards achieving the desired coupling to the two sublattices. While Fig. 4 depicts an example of coupling two normal metals to the antiferromagnetic sublattices, the general objective is to couple the two systems to be entangled, which are not necessarily metals, to the opposite sublattices.
[1] C. Gerry and P. Knight, Introductory Quantum Optics (Cambridge University Press, Cambridge, 2004).
[2] R. Schnabel, Squeezed states of light and their applications in laser interferometers, Phys. Rep. 684, 1 (2017). 
[3] B. P. Abbott et al. (LIGO Scientific Collaboration and Virgo Collaboration), Observation of Gravitational Waves from a Binary Black Hole Merger, Phys. Rev. Lett. 116, 061102 (2016).

[4] The LIGO Scientific Collaboration, A gravitational wave observatory operating beyond the quantum shot-noise limit, Nat. Phys. 7, 962 (2011).

[5] The LIGO Scientific Collaboration, Enhanced sensitivity of the LIGO gravitational wave detector by using squeezed states of light, Nat. Photonics 7, 613 (2013).

[6] H. Grote, K. Danzmann, K. L. Dooley, R. Schnabel, J. Slutsky, and H. Vahlbruch, First Long-Term Application of Squeezed States of Light in a Gravitational-Wave Observatory, Phys. Rev. Lett. 110, 181101 (2013).

[7] Z. Y. Ou, S. F. Pereira, H. J. Kimble, and K. C. Peng, Realization of the Einstein-Podolsky-Rosen Paradox for Continuous Variables, Phys. Rev. Lett. 68, 3663 (1992).

[8] T. C. Ralph, Continuous variable quantum cryptography, Phys. Rev. A 61, 010303(R) (1999).

[9] G. J. Milburn and S. L. Braunstein, Quantum teleportation with squeezed vacuum states, Phys. Rev. A 60, 937 (1999).

[10] F. Furrer, T. Franz, M. Berta, A. Leverrier, V. B. Scholz, M. Tomamichel, and R. F. Werner, Continuous Variable Quantum Key Distribution: Finite-Key Analysis of Composable Security Against Coherent Attacks, Phys. Rev. Lett. 109, 100502 (2012).

[11] A. Eddins, S. Schreppler, D. M. Toyli, L. S. Martin, S. HacohenGourgy, L. C. G. Govia, H. Ribeiro, A. A. Clerk, and I. Siddiqi, Stroboscopic Qubit Measurement with Squeezed Illumination, Phys. Rev. Lett. 120, 040505 (2018).

[12] P. Král, Displaced and squeezed Fock states, J. Mod. Opt. 37, 889 (1990).

[13] M. M. Nieto, Displaced and squeezed number states, Phys. Lett. A 229, 135 (1997).

[14] J. Zhao, A. V. Bragas, D. J. Lockwood, and R. Merlin, Magnon Squeezing in an Antiferromagnet: Reducing the Spin Noise Below the Standard Quantum Limit, Phys. Rev. Lett. 93, 107203 (2004).

[15] J. Zhao, A. V. Bragas, R. Merlin, and D. J. Lockwood, Magnon squeezing in antiferromagnetic $\mathrm{MnF}_{2}$ and $\mathrm{FeF}_{2}$, Phys. Rev. B 73, 184434 (2006).

[16] D. Bossini, S. D. Conte, Y. Hashimoto, A. Secchi, R. V. Pisarev, T. Rasing, G. Cerullo, and A. V. Kimel, Macrospin dynamics in antiferromagnets triggered by sub-20 femtosecond injection of nanomagnons, Nat. Commun. 7, 10645 (2016).

[17] D. Bossini, S. Dal Conte, G. Cerullo, O. Gomonay, R. V. Pisarev, M. Borovsak, D. Mihailovic, J. Sinova, J. H. Mentink, T. Rasing, and A. V. Kimel, Laser-driven quantum magnonics and terahertz dynamics of the order parameter in antiferromagnets, Phys. Rev. B 100, 024428 (2019).

[18] S. L. Johnson, P. Beaud, E. Vorobeva, C. J. Milne, É. D. Murray, S. Fahy, and G. Ingold, Directly Observing Squeezed Phonon States with Femtosecond X-Ray Diffraction, Phys. Rev. Lett. 102, 175503 (2009).

[19] A. Kamra and W. Belzig, Super-Poissonian Shot Noise of Squeezed-Magnon Mediated Spin Transport, Phys. Rev. Lett. 116, 146601 (2016).

[20] A. Kamra, U. Agrawal, and W. Belzig, Noninteger-spin magnonic excitations in untextured magnets, Phys. Rev. B 96, 020411(R) (2017).
[21] A. Kamra and W. Belzig, Magnon-mediated spin current noise in ferromagnet | nonmagnetic conductor hybrids, Phys. Rev. B 94, 014419 (2016).

[22] The "spin-1" magnon is a quasiparticle that carries a spin of $\hbar$ along the $z$ direction [42]. It is not an actual $S=1$ bosonic particle.

[23] Within the adopted terminology convention, if one were to generate a nonequilibrium squeezed state of spin excitations in an anisotropic ferromagnet, it would be called the "squeezed state of squeezed magnons."

[24] E. Saitoh, M. Ueda, H. Miyajima, and G. Tatara, Conversion of spin current into charge current at room temperature: Inverse spin-Hall effect, Appl. Phys. Lett. 88, 182509 (2006).

[25] X. He, Y. Wang, N. Wu, A. N. Caruso, E. Vescovo, K. D. Belashchenko, P. A. Dowben, and C. Binek, Robust isothermal electric control of exchange bias at room temperature, Nat. Mater. 9, 579 (2010).

[26] W. Zhang and K. M. Krishnan, Epitaxial exchange-bias systems: From fundamentals to future spin-orbitronics, Mater. Sci. Eng., R 105, 1 (2016).

[27] P. Wadley, B. Howells, J. Železný, C. Andrews, V. Hills, R. P. Campion, V. Novák, K. Olejník, F. Maccherozzi, S. S. Dhesi, S. Y. Martin, T. Wagner, J. Wunderlich, F. Freimuth, Y. Mokrousov, J. Kuneš, J. S. Chauhan, M. J. Grzybowski, A. W. Rushforth, K. W. Edmonds, B. L. Gallagher, and T. Jungwirth, Electrical switching of an antiferromagnet, Science 351, 587 (2016).

[28] T. Kosub, M. Kopte, R. Hühne, P. Appel, B. Shields, P. Maletinsky, R. Hübner, M. O. Liedke, J. Fassbender, O. G. Schmidt, and D. Makarov, Purely antiferromagnetic magnetoelectric random access memory, Nat. Commun. 8, 13985 (2017).

[29] R. Lebrun, A. Ross, S. A. Bender, A. Qaiumzadeh, L. Baldrati, J. Cramer, A. Brataas, R. A. Duine, and M. Kläui, Tunable longdistance spin transport in a crystalline antiferromagnetic iron oxide, Nature (London) 561, 222 (2018).

[30] E. V. Gomonay and V. M. Loktev, Spintronics of antiferromagnetic systems (review article), Low Temp. Phys. 40, 17 (2014).

[31] T. Jungwirth, X. Marti, P. Wadley, and J. Wunderlich, Antiferromagnetic spintronics, Nat. Nanotechnology 11, 231 (2016).

[32] O. Gomonay, V. Baltz, A. Brataas, and Y. Tserkovnyak, Antiferromagnetic spin textures and dynamics, Nat. Phys. 14, 213 (2018).

[33] V. Baltz, A. Manchon, M. Tsoi, T. Moriyama, T. Ono, and Y. Tserkovnyak, Antiferromagnetic spintronics, Rev. Mod. Phys. 90, 015005 (2018).

[34] L. Šmejkal, Y. Mokrousov, B. Yan, and A. H. MacDonald, Topological antiferromagnetic spintronics, Nat. Phys. 14, 242 (2018).

[35] S. Sachdev, Quantum Phase Transitions (Cambridge University Press, Cambridge, 2001).

[36] C. Castelnovo, R. Moessner, and S. L. Sondhi, Spin liquids in frustrated magnets, Nature (London) 451, 42 (2008).

[37] L. Balents, Spin liquids in frustrated magnets, Nature (London) 464, 199 (2010).

[38] L. Savary and L. Balents, Quantum spin liquids: A review, Rep. Prog. Phys. 80, 016502 (2017).

[39] T. Nishioka, Entanglement entropy: Holography and renormalization group, Rev. Mod. Phys. 90, 035007 (2018). 
[40] G. P. Rodrigue, H. Meyer, and R. V. Jones, Resonance measurements in magnetic garnets, J. Appl. Phys. 31, S376 (1960).

[41] L. Liensberger, A. Kamra, H. Maier-Flaig, S. Geprägs, A. Erb, S. T. B. Goennenwein, R. Gross, W. Belzig, H. Huebl, and M. Weiler, Exchange-Enhanced Ultrastrong Magnon-Magnon Coupling in a Compensated Ferrimagnet, Phys. Rev. Lett. 123, 117204 (2019).

[42] C. Kittel, Quantum Theory of Solids (Wiley, New York, 1963).

[43] T. Holstein and H. Primakoff, Field dependence of the intrinsic domain magnetization of a ferromagnet, Phys. Rev. 58, 1098 (1940).

[44] A. I. Akhiezer, V. G. Bar'iakhtar, and S. V. Peletminski, Spin Waves (North-Holland, Amsterdam, 1968).

[45] We assume $C_{\boldsymbol{k}}$ is positive.

[46] In defining the squeeze operator, we have implicitly assumed positive $C_{k}$. If $C_{k}$ is negative, we obtain the same non-negative squeeze parameter with a squeezing phase of $\pi$ [1]. The phenomena studied herein remain unaffected under such a phase shift.

[47] H. Vahlbruch, M. Mehmet, K. Danzmann, and R. Schnabel, Detection of $15 \mathrm{db}$ Squeezed States of Light and their Application for the Absolute Calibration of Photoelectric Quantum Efficiency, Phys. Rev. Lett. 117, 110801 (2016).

[48] P. K. Manna and S. M. Yusuf, Two interface effects: Exchange bias and magnetic proximity, Phys. Rep. 535, 61 (2014).

[49] A. Kamra and W. Belzig, Spin Pumping and Shot Noise in Ferrimagnets: Bridging Ferro- and Antiferromagnets, Phys. Rev. Lett. 119, 197201 (2017).

[50] A. Kamra, A. Rezaei, and W. Belzig, Spin Splitting Induced in a Superconductor by an Antiferromagnetic Insulator, Phys. Rev. Lett. 121, 247702 (2018).

[51] E. Erlandsen, A. Kamra, A. Brataas, and A. Sudbø, Enhancement of superconductivity mediated by antiferromagnetic squeezed magnons, Phys. Rev. B 100, 100503(R) (2019).

[52] J. Zou, S. K. Kim, and Y. Tserkovnyak, Tuning entanglement by squeezing magnons in anisotropic magnets, arXiv:1909.09653.

[53] L. J. Cornelissen, J. Liu, R. A. Duine, J. B. Youssef, and B. J. van Wees, Long-distance transport of magnon spin information in a magnetic insulator at room temperature, Nat. Phys. 11, 1022 (2015).

[54] S. T. B. Goennenwein, R. Schlitz, M. Pernpeintner, K. Ganzhorn, M. Althammer, R. Gross, and H. Huebl, Non-local magnetoresistance in YIG/Pt nanostructures, Appl. Phys. Lett. 107, 172405 (2015).
[55] S. A. Bender, A. Kamra, W. Belzig, and R. A. Duine, Spin Current Cross-Correlations as a Probe of Magnon Coherence, Phys. Rev. Lett. 122, 187701 (2019).

[56] Ø. Johansen, A. Kamra, C. Ulloa, A. Brataas, and R. A. Duine, Magnon-Mediated Indirect Exciton Condensation through Antiferromagnetic Insulators, Phys. Rev. Lett. 123, 167203 (2019).

[57] D. MacNeill, J. T. Hou, D. R. Klein, P. Zhang, P. Jarillo-Herrero, and L. Liu, Gigahertz Frequency Antiferromagnetic Resonance and Strong Magnon-Magnon Coupling in the Layered Crystal $\mathrm{CrCl}_{3}$, Phys. Rev. Lett. 123, 047204 (2019).

[58] Strictly speaking, second-order Rényi entropy, which provides a lower bound on von Neumann entropy, was measured.

[59] R. Islam, R. Ma, P. M. Preiss, M. E. Tai, A. Lukin, M. Rispoli, and M. Greiner, Measuring entanglement entropy in a quantum many-body system, Nature (London) 528, 77 (2015).

[60] A. Kamra, R. E. Troncoso, W. Belzig, and A. Brataas, Gilbert damping phenomenology for two-sublattice magnets, Phys. Rev. B 98, 184402 (2018).

[61] C. Leroux, L. C. G. Govia, and A. A. Clerk, Enhancing Cavity Quantum Electrodynamics via Antisqueezing: Synthetic Ultrastrong Coupling, Phys. Rev. Lett. 120, 093602 (2018).

[62] W. Qin, A. Miranowicz, P.-B. Li, X.-Y. Lü, J. Q. You, and F. Nori, Exponentially Enhanced Light-Matter Interaction, Cooperativities, and Steady-State Entanglement Using Parametric Amplification, Phys. Rev. Lett. 120, 093601 (2018).

[63] R. J. Glauber, The quantum theory of optical coherence, Phys. Rev. 130, 2529 (1963).

[64] E. C. G. Sudarshan, Equivalence of Semiclassical and Quantum Mechanical Descriptions of Statistical Light Beams, Phys. Rev. Lett. 10, 277 (1963).

[65] I. Bloch, J. Dalibard, and W. Zwerger, Many-body physics with ultracold gases, Rev. Mod. Phys. 80, 885 (2008).

[66] V. Galitski and I. B. Spielman, Spin-orbit coupling in quantum gases, Nature (London) 494, 49 (2013).

[67] V. Galitski, G. Juzeliūnas, and I. B. Spielman, Artificial gauge fields with ultracold atoms, Phys. Today 72(1), 38 (2019).

[68] Quadrature squeezing, however, comments on the ellipticity in the quantum fluctuations and not the expectation values of the spins in the coherent state, as is the case with the ellipticity in the classical spin wave picture. The two kinds of ellipticities, although interrelated in equilibrium for the case under discussion, do not need to be identical in general.

[69] In the following discussion, we are concerned with the $z$ projected spin without specifying this directional preference explicitly. 